\title{
APLICAÇÃO DOS SISTEMAS MISO E SIMO NAS COMUNICAÇÕES MÓVEIS
}

\author{
APPLICATION OF TWO SYSTEMS MISO E SIMO NAS COMUNICAÇÕES MÓVEIS
}

\author{
Douglas Kleiton Souza Magalhães ${ }^{1}$ \\ Jeorkley Gomes Silva ${ }^{2}$ \\ Maçuete Austriaco Teixeira Neto ${ }^{3}$ \\ Matheus Carvalho Reis ${ }^{4}$ \\ Jonathan Araújo Queiroz ${ }^{5}$
}

RESUMO: Esta pesquisa versa sobre os sistemas MISO e SIMO, cujo objetivo geral consistiu em compreender a funcionalidade desses sistemas em vista da comunicação sem fio, a partir de uma perspectiva comparativa entre os referidos sistemas, levando em consideração as suas especificidades, vantagens e desvantagens. Para isso, a metodologia adotada se fundamentou em uma pesquisa bibliográfica, a partir de consulta em livros, revistas, periódicos, dissertações e artigos científicos que foram levantados nas bases de dados LILACS e Scielo, caracterizando-se, portanto, como uma pesquisa de natureza qualitativa e exploratória, com abordagem descritiva. Verificou-se que as antenas inteligentes que abrangem a tecnologia dos sistemas MISO e SIMO são ferramentas eficazes para resolver problemas com desvios e pouca abrangência na transmissão de dados durante a comunicação. Conclui-se que o uso de antenas múltiplas tanto na transmissão quanto na recepção traz mais dinamicidade e modernidade no âmbito das comunicações sem fio e benefícios para os usuários como diminuição no desgaste da bateria dos aparelhos móveis e redução no nível de interferência e desvios na comunicação.

Palavras-chave: Sistemas. SIMO. MISO. Antenas Inteligentes.

ABSTRACT: This research deals with the MISO and SIMO systems, whose general objective consisted in understanding the functionality of these systems in view of non-wired communication, from a comparative perspective between the aforementioned systems, considering their specificities, advantages and disadvantages. For this, the methodology adopted is based on a bibliographical research, from consultation in books, magazines, newspapers, dissertations and scientific articles that were raised in the LILACS and Scielo databases, characterizing itself, therefore, as a research of nature qualitative and exploratory, with a descriptive approach. I verified that the smart antennas that embrace the technology of both MISO and SIMO systems are effective tools to solve problems with deviations and poor coverage in data transmission during communication. I concluded that the use of multiple antennas, both in transmission and reception, is more dynamic and modern in the field of non-fiber communication and benefits for users such as a reduction in the use of the battery of mobile devices and a reduction in the level of interference and deviations in communication.

Keywords: Systems. SIMO. MISO. Smart Antennas.

\footnotetext{
Graduando em Engenharia de Computação na Universidade Ceuma - E-mail: douglaskleitoni@gmail.com ${ }^{2}$ Graduando em Engenharia de Computação na Universidade Ceuma-E-mail: jeankleysilva@hotmail.com

3 Graduando em Engenharia de Computação na Universidade Ceuma - E-mail: massueteaustriaco@gmail.com

${ }^{4}$ Graduando em Engenharia de Computação na Universidade Ceuma - E-mail: matheusreis.mr4@gmail.com

${ }^{5}$ Professor orientador. Universidade Ceuma-E-mail: janathan.queiroz@ceuma.com.br.
} 


\section{INTRODUÇÃO}

A sociedade contemporânea tem presenciado diversas mudanças, sobretudo, pelo avanço tecnológico, que a partir da inserção das Tecnologias da Informação e Comunicação (TICs) denotou uma ruptura nos padrões convencionais em diversas esferas da sociedade. É notório que na atualidade o acesso às TICs tornou-se uma realidade comum no cotidiano dos indivíduos, devido ao barateamento dessas tecnologias que implicou na ampliação do acesso a essas tecnologias por diferentes classes sociais, citando como exemplo, o uso de aparelhos móveis celulares que se tornou uma ferramenta indispensável na vida das pessoas.

Todavia, essa facilidade também implicou em problemas, pois com o aumento exponencial do compartilhamento de informações em tempo real, algumas tecnologias de transmissão de dados que antes eram empregadas para esse fim, caíram em desuso ou então passaram a operar com pouca eficiência.

Nesse contexto, se destaca a crescente procura na atualidade por transmissão de dados em sistemas de comunicação móvel, que se sobrepõe de maneira positiva ao modelo convencional de comunicação, por melhorar a velocidade dos dados e por utilizar múltiplas antenas que diminuem os desvios e ruídos na comunicação, além de maximizar a capacidade de transmissão, características essas que são próprias de sistemas mais dinâmicos e modernos, denominados de sistemas MISO, SIMO e MIMO.

Para essa pesquisa, a linha de estudo abrangeu apenas os sistemas MISO e SIMO, também conhecidos como produtos da tecnologia das antenas inteligentes que se baseiam na utilização de múltiplas antenas que estão conectadas ao mesmo dispositivo. Desta forma, o seu objetivo geral consistiu em compreender a funcionalidade desses sistemas em vista da comunicação sem fio, a partir de uma perspectiva comparativa, a fim de conhecer as especificidades destes sistemas, vantagens e desvantagens.

Os procedimentos metodológicos adotados tiveram por base uma pesquisa bibliográfica com consulta em livros, revistas, periódicos, dissertações, artigos científicos, dentre outros aportes teóricos e foram levantados nas bases de dados LILACS e Scielo, caracterizando-se, portanto, como uma pesquisa de natureza qualitativa e exploratória, com abordagem descritiva. 
Para o alcance do objetivo geral e discussão da temática, estruturou-se esse estudo em 4 (quatro) capítulos, incluindo este capítulo da introdução.

No primeiro capítulo abordaram-se aspectos gerais sobre a definição e características da tecnologia das antenas inteligentes que abrange os sistemas MISO e SIMO; no segundo capítulo apresentaram-se os resultados da pesquisa com base no objetivo proposto e a estruturação da metodologia adotada.

No quarto e último capítulo realizou-se considerações acerca dos resultados alcançados, além de outras informações.

\section{ASPECTOS GERAIS DA TECNOLOGIA DE ANTENA INTELIGENTE}

Nos últimos anos, ocorreram diversas mudanças na sociedade como um todo, devidas especialmente ao avanço tecnológico (PORTAL EDUCAÇÃO, 2013). Tais transformações tem desempenhado um papel inovador nos aspectos socioeconômico, político e cultural, e as Tecnologias da Informação e Comunicação (TICs) estão entre essas grandes transformações, cuja principal característica reside na redução do tempo de comunicação e nas diversas possibilidades de interação, que são produtos da era digital que instituiu um novo padrão devido ao advento da internet (PISCHETOLA, 2019).

Nesse contexto, se destaca a transmissão de informação que é executada no sistema de comunicação sem fio, que utiliza ondas eletromagnéticas para realizar o processo de comunicação, citando como exemplo, os padrões abrangidos na família 802 do Institute of Electrical and Electronics Engineers (IEEE), sendo tais: Wireless LAN e Mesh, Wireless PAN, Certificação Bluetooth, Coexistência do IEEE 802.15 e 803.II, High-Rate wireless PAN, Broadband Wireless Access, dentre outros; esses padrões denotam um grande avanço no que tange às diversas possibilidades de troca de informações (RAMOS, 2016).

Sistemas são definidos como componentes físicos ou algoritmos que executam o sinal de saída por meio do sinal de entrada, sendo os sinais um conjunto de informações ou dados que são executados por esses sistemas, visando modificar estes sinais ou descobrir informações específicas, portanto, os sistemas são reconhecidos pelas suas entradas, saídas e pelas regras de operação que os controlam (LATHI, 1998).

Destacando-se as regras de operação, estas podem ser representadas a partir de equações matemáticas com base no conhecimento em áreas específicas e algumas medições (PROHMANN, 2018). As formas de representar essas equações assentam-se no espaço de 
estados e nas equações diferenciais lineares ou não lineares de primeira ordem, quando se tratar de sistemas dinâmicos, ou seja, sistemas cujas saídas atuais dependem dos valores que foram passados na entrada (LATHI, I998).

Ilustra-se na figura I uma estrutura simplificada de um sistema.

Figura I - Diagrama de um sistema

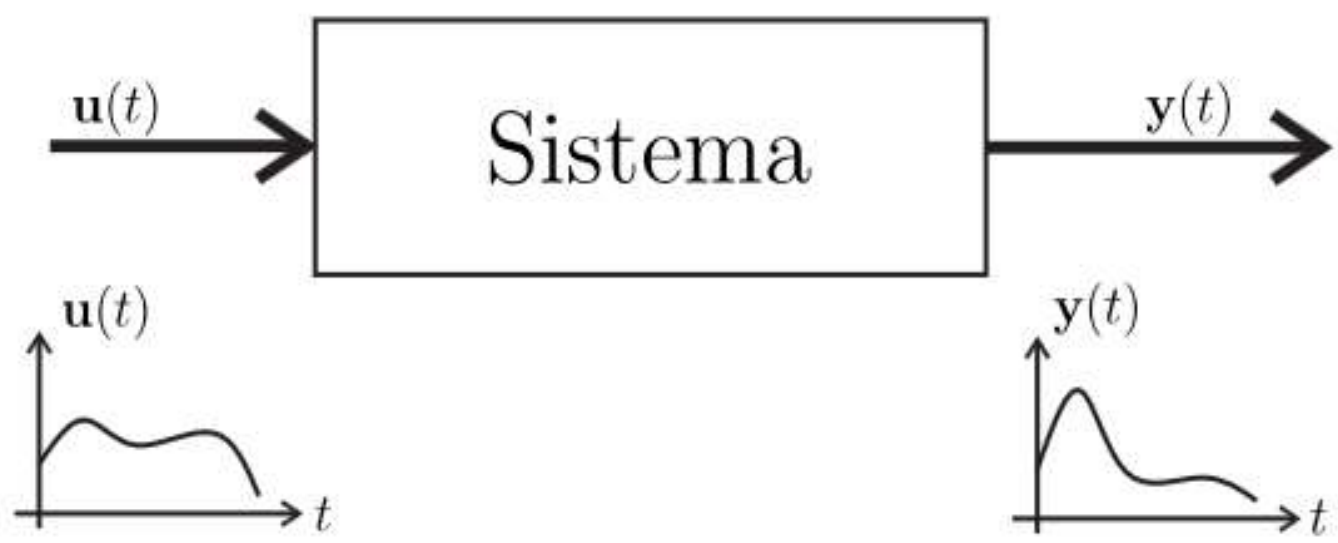

Fonte: Prohmann (2018, p. 22)

Desta forma, o $\mathbf{u}(\mathrm{t})$ representa o sinal de entrada do sistema e o $\mathbf{y}(\mathrm{t})$ equivale ao sinal que foi gerado na saída do sistema (PROHMANN, 2018).

A procura por serviços de internet, televisão de alta definição e serviços de multimídia está em constante evolução e a demanda por estes serviços está cada dia mais comum, isso implica na necessidade de se criar tecnologias com maior abrangência para implementar esses serviços de maneira eficiente (ALMEIDA; MOTA; ROCHA, 20I0).

A demanda por transmissão de dados em sistemas móveis na contemporaneidade cresceu significativamente a taxas muito elevadas, e o setor industrial prevê que a dilatação do volume de tráfego será exponencialmente maior nos próximos anos (CHEN; ZHAO, 2014).

Conforme dados de uma pesquisa, a previsão de crescimento de tráfego e de equipamentos que estarão conectados a redes Internet Protocol até o ano de 2020 será em média 2,3 ZB e 3,4 vezes o número de habitantes (CISCO, 2016).

Demonstra-se na Figura 2 um exemplo de interação e compartilhamento de informações de comunicação sem fio. 
Figura 2 - Troca de informações entre tecnologias

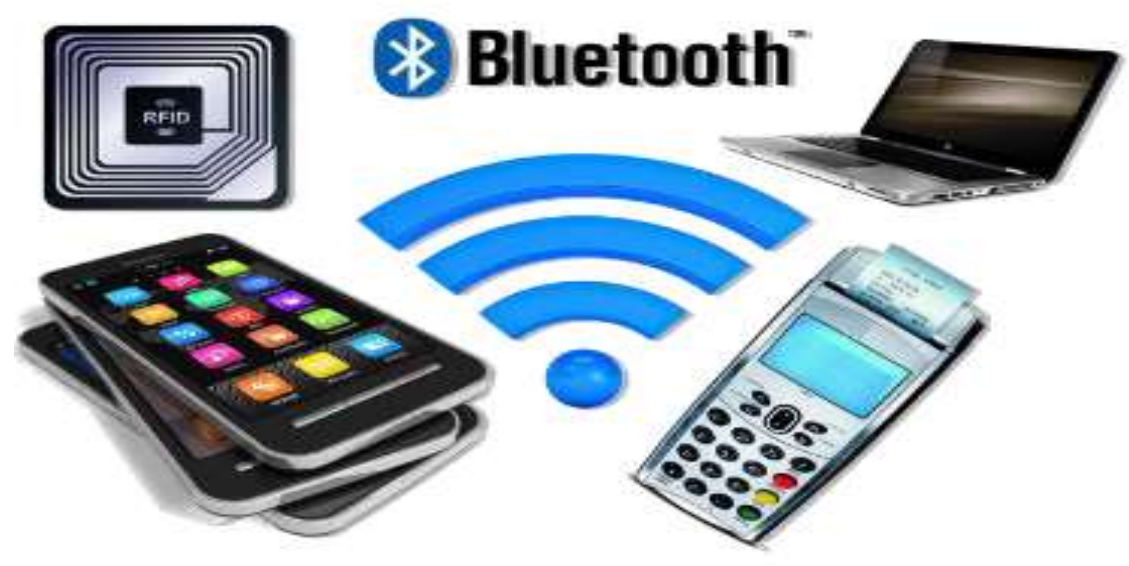

Fonte: Ramos (2016, p. 21).

Os sistemas para comunicação móveis sem fio, bem como exemplificados na Figura 2, são executados pela tecnologia Multiple Input Multiple Outputs (MIMO), que consiste em um sistema que utiliza diversas antenas conectadas ao mesmo dispositivo, e que interagem mutuamente com o intuito de diminuir erros e melhorar a velocidade dos dados e a capacidade de transmissão dos mesmos, configurando-se como as principais vantagens desse sistema, além de reduzir interrupções; com isso, os dispositivos que estiverem conectados ao roteador levarão menor tempo para receber dados (ANLIX, 2018).

Embora isso, a utilização de múltiplas antenas conectadas entre si apresente alguns problemas, como por exemplo, a emissão de radiações eletromagnéticas no espaço, uma vez que quando se faz uso dessas desse tipo de antena, uma parte do sinal transmitido pode irradiar para regiões indesejadas e prejudicar o sinal de outras tecnologias provocando ruídos (RAMOS, 2016).

Ainda nesse aspecto, o sistema MIMO não é a solução mais apropriada para situações cotidianas, pois ele precisa de um sistema mais corpulento e mais complexo para atender às demandas esperadas, por outro lado, trata-se de um sistema muito simples no que diz respeito a sua implementação, representa ainda um caminho propício para o aumento da eficiência espectral a partir das antenas inteligentes (REDDY; BHALCHANDRA; RATNAPARKHE, 2014).

Uma solução para corrigir tais desvios, tornando os equipamentos de transmissão mais eficientes, reside no emprego de antenas inteligentes, as smart antennas, que usam 
diagramas de irradiação para corrigir e controlar de maneira eletrônica (SAFARI; SHAFAI C.; SHAFAI L, 2015). Desta forma, a compreensão sobre a utilização de antenas no transmissor e no receptor como recurso para potencializar o seu desempenho, perpassa pela abordagem dos sistemas MIMO, Single Outpout (SISO), Single Input Multiple Outpout (SIMO) e Multiple Input Single (MISO) (CISCO, 2016).

A lógica de uma antena inteligente é usar a informação disponível por meio dos vários elementos da rede de antenas, que se for devidamente processada, possibilita a adaptação do diagrama de radiação a partir do controle de amplitudes e fases para a direção desejada, e para isso, é necessário que se utilize um sinal de treinamento e a direção de chegada desse sinal (SOUSA, 2015).

A aplicação de antenas inteligentes em sistemas de comunicação tem se tornado um modelo importante no que diz respeito ao desenvolvimento de sistemas de comunicação sem fio em ascensão; essa técnica tem se mostrado como uma alternativa para resolver os desafios na atualidade, citando como exemplo, o alcance de elevada taxa de transmissão e um significativo aumento na qualidade dos sistemas de comunicação, motivo pelo qual diversas indústrias têm desenvolvido com muita frequência, produtos que fazem uso das smart antenas (SALAMANCA, 2012).

\section{RESULTADOS DA PESQUISA 3.I Metodologia da pesquisa}

O presente artigo versou sobre a temática "aplicabilidade dos sistemas MISO e SIMO”, cujo objetivo geral consistiu em fazer uma compreender como se dá a aplicação desses sistemas em face da comunicação móvel, levando em consideração as suas especificidades, vantagens e desvantagens dos mesmos. Em vista disso, esse estudo se caracteriza como uma pesquisa básica, descritiva, exploratória e com abordagem qualitativa; quanto aos procedimentos adotados na coleta de dados, trata-se de uma pesquisa bibliográfica.

A pesquisa básica e exploratória é definida por Barros e Lehfeld (2014) como aquela que visa atualizar os conhecimentos do pesquisador sobre determinado fenômeno, ou seja, no primeiro momento não há a pretensão de interferir na realidade dos fatos, mas somente satisfazer uma necessidade intelectual, explorando assim a natureza dos fatos para ampliar o conhecimento. 
Esta pesquisa é descritiva por apontar características de uma determinada população, estabelecendo conexões entre as variáveis correspondentes (VERGARA, 20II). A pesquisa qualitativa se dá a partir da conexão entre uma relação dinâmica com o mundo real, considerando o pesquisador que investiga os fatos que compõem essa realidade, onde tais relações não seriam possíveis em uma pesquisa de natureza quantitativa (FILHO; FILHO, 2013).

A pesquisa descritiva consiste em fornecer exemplos e situações análogas, mas sem a intenção de abranger todos os fatos (STAKE, 20II). Assim sendo, buscou-se conhecer as especificidades dos sistemas MISO E SIMO, a fim de e compreender como esses sistemas são operacionalizados, as suas principais vantagens e desvantagens, a nexo comparativo entre os mesmos.

O levantamento bibliográfico foi realizado a partir da consulta em livros, revistas especializadas, artigos científicos, dissertações, periódicos, dentre outros aportes. A coleta de informações foi feita nas bases de dados da Literatura Latino - Americana e do Caribe (LILACS) e Biblioteca Científica Eletrônica Online (Scielo).

\subsection{Especificidades e comparação entre os sistemas SIMO e MISO}

Os sistemas de comunicação móveis tradicionais se baseiam no uso de uma única antena de transmissão e recepção, caracterizando-se como sistema SISO; nesse tipo de modelagem são explorados os domínios do tempo, frequência e codificação (ALMEIDA; MOTA; ROCHA, 2010). Apresenta-se na Figura 3 um modelo de sistema de comunicação móvel tradicional, definido como sistema SISO.

Figura 3 - Sistema SISO
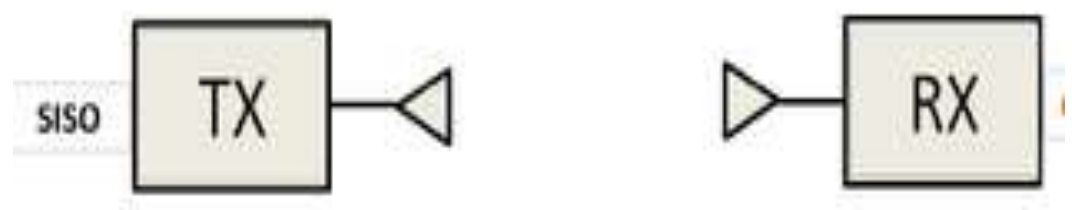

Fonte: Adaptado de Martins Jr (20II).

Todavia, por fazer uso apenas de uma antena tanto para transmitir quanto para receber o sinal e tendo em vista a crescente demanda por serviços multimídia, é necessário 
que ocorra um aumento na taxa de transmissão de dados para garantir maior confiabilidade aos sistemas, o que faz com que o SISO não seja o sistema mais indicado para algumas realidades, como por exemplo, nos sistemas de comunicação sem fio (MARTINS JR, 20II).

Nos sistemas MISO e SIMO, um dos seus terminais encontra-se equipado por um conjunto de antenas que promovem a exploração parcial do domínio espacial, implicando em maiores ganhos na diversidade espacial e na técnica de formação de feixe (ALMEIDA; MOTA; ROCHA, 2010). Podem ainda ser adaptados em redes de computadores para serem usados nos terminais móveis, logo, podem ser aplicados em ambiente civil, militar e comercial (SCHLUB; LU; OHIRA, 2003).

O esquema de transmissão e recepção do sinal por múltiplas antenas pode ser visto a partir da Figura 4.

Figura 4 - Sistemas MISO e SIMO
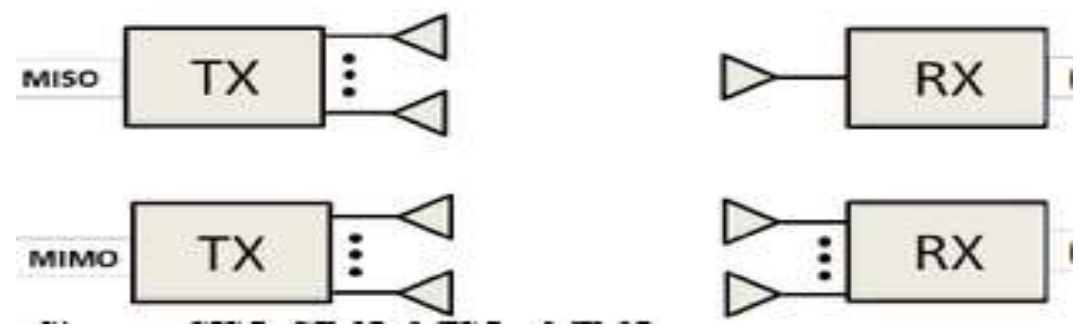

Fonte: Adaptado de Martins Jr (20II).

Os sistemas MISO e SIMO utilizam a técnica de diversidade espacial, que se fundamenta no envio da informação por diferentes antenas, cuja eficácia depende dos enlaces que são formados por diferentes pares de antenas, sendo uma transmissora e a outra receptora, possibilitando que o código que foi enviado por meios desses enlaces alcance diferentes desvanecimentos (KYM et al.; 2008).

O SIMO utiliza uma antena de transmissão e duas antenas de recepção, o que faz com que a taxa de transmissão de dados alcance melhores resultados quando comparado ao sistema SISO, no SIMO existe uma combinação dos sinais que resultaram da conexão entre as duas antenas, possibilitando que a estação escolha o melhor dos dois sinais e assim melhore a qualidade do seu sinal (BROWN; KYRITSI; CARVALHO, 2012).

Martins Jr (20II) explica que o plano de fundo do sistema SIMO consiste em: aumentando-se a quantidade de antenas na recepção, a capacidade do canal de comunicação 
também se expande; enquanto que o sistema MISO funciona com múltiplas antenas na transmissão, apresentando assim uma diversidade na transmissão do sinal com várias antenas; aumentando-se a quantidade de antenas transmissoras, aumenta significativamente a capacidade do canal de comunicação.

Em síntese, as principais vantagens dos sistemas MISO e SIMO residem em maior incremento da zona de cobertura, pois o sinal é recebido a uma menor distancia; redução da potência transmitida e consequentemente o aumento da sensibilidade da Estação Rádio Base (ERB), que implica na diminuição na potência nos equipamentos dos usuários de dispositivos móveis, economizando a bateria destes dispositivos; diminuição da propagação multipercurso, pois com uma menor dispersão angular da potência da ERB, se reduziria os múltiplos percursos até alcançar o usuário móvel; reduz o nível de interferência, pois a antena seleciona o sinal correto evitando que outros sinais interfiram na comunicação (ALMEIDA; MOTA; ROCHA, 20I0).

De maneira geral, uma das desvantagens quanto à utilização dos sistemas supracitados, refere-se ao desperdício de potência, pois o sinal é transmitido para direções onde não existe nenhuma espécie de terminal móvel para recebê-lo, além de que o sinal que é transmitido para todas as direções, pode causar interferências em outros tipos de terminais móveis (PER; PETTERSON, 1999).

\section{CONSIDERAÇÕES FINAIS}

Esta pesquisa que abordou sobre a temática "aplicação dos sistemas SIMO e MISO em sistemas de comunicações móveis”, alcançou o objetivo proposto que foi o de compreender as especificidades, vantagens e desvantagens desses sistemas em situações cotidianas, pois como visto durante a construção teórica, ambos os sistemas fazem uso da técnica da diversidade espacial por meio do compartilhamento do sinal por múltiplas antenas, que trazem benefícios significativos tanto no que diz respeito à capacidade de transmissão de dados, quanto na capacidade de recepção dos mesmos, além da mitigação dos ruídos na comunicação.

E em vista da atual configuração social que demanda por meios mais modernos e dinâmicos de comunicação, estes sistemas representam uma alternativa viável e eficaz para resolver problemas como desvios e pouca abrangência na transmissão de dados durante a comunicação. 
Outro aspecto relevante que afeta o usuário de maneira positiva, é que o uso dos sistemas SIMO e MISO nas tecnologias móveis, mais especificamente o aparelho celular, contribui com a vida útil da bateria devido à diminuição na potência neste equipamento, implicando assim em maior economia para o usuário.

Nesta pesquisa enfatizou-se a aplicação dos sistemas SIMO e MISO e redes móveis de comunicação, mas recomenda-se que se amplie o estudo desta temática a partir de outras perspectivas, como por exemplo, sobre questões relacionadas aos benefícios que estes sistemas trazem para a vida dos usuários.

\section{REFERÊNCIAS}

ANLIX. Tecnologia MIMO no roteador: o que é e como funciona? 2018. Disponível em: https://anlix.io/tecnologia-mimo-no-roteador-o-que-e-e-como-funciona/. Acesso em: 22 jan. 2022.

BARROS, A. J. da S; LEHFELD, N. A. de S. Fundamentos de Metodologia Científica. 3. ed. São Paulo: Pearson Prentice Hall, 2014.

BROWN, T.; KYRITSI, P.; CARVALHO, E. de. Practical Guide to the MIMO Radio channel with MATLAB. United Kingdom: John Wiley \& Sons, 2012.

KIM, Y. et al. Compact Built-In Handset MIMO Antenna Using L-Shaped Folded Monopole Antennas. 2008. IEICE TRANSACTIONS on Communications, v. E91-B, n. 6, p.I743-I75I. Disponível em: https://search.ieice.org/bin/summary.php?id=e9I-b_6_I743. Acesso em: 20 jan. 2022.

LATHI, B. P. Signal processing and linear systems. Oxford university press New York, 1998.

PEH, H. L.; PETTERSON, Magne. "An Overview of Smart Antennas Technology for Mobile Communications Systems". IEEE Communications - Fourth Quarter, v. 2, n. 4, I999.

PROHMANN, E. A. V. Proposta de um Filtro de Partículas Aliado ao Filtro de Kalman Estendido Iterativo para Estimação de Estados de Sistemas Não Lineares com Ruído Gaussiano. 2018. II4 p. Dissertação (Programa de Pós-Graduação em Engenharia de Eletricidade) - Universidade Federal do Maranhão, 2018.

REDDY, B. S.; BHALCHANDRA, A. S.; RATNAPARKHE, V. R. Adaptive Digital Beam Forming using LMS Algorithm Aurangabad, India: Iosr Journal Of Electronics And Communication Engineering (iosr-jece), 2014.

SAFARI M.; SHAFAI C.; SHAFAI L. "X-Band Tunable Frequency Selective Surface Using MEMS Capacitive Loads,”. IEEE Transactions on Antennas and Propagation, v. 3. 2015. 
Disponível em: file://C:/Users/pc/AppData/Local/Temp/TAP.2014.2386304.pdf. Acesso em: 21 jan. 2022.

SALAMANCA, J. J. L. Utilização Conjunta de Técnicas de Formatação de Feixe, Diversidade Espacial e Equalização na Recepção de Sinais. Florianópolis: Universidade Federal de Santa Catarina, 2012.

SCHLUB, R.; LU, J.; OHIRA, T. "Seven-Element Ground Skirt Monopole ESPAR Antenna Design from a Genetic Algorithm and the Finite Element Method,". IEEE Transactions on Antennas and Propagation, v. 5I, n. II, p. 3033-3039, 2003.

SOUSA, Í. A. do N. Implementação de filtragem espacial adaptativa para sistemas de recepção com múltiplas antenas. $2015.58 \mathrm{p}$. (Monografia) - Universidade de Brasília - UnB, Brasília, DF, 2015. Disponível em: https://bdm.unb.br/bitstream/10483/14063/I/2015_ItaloAlbertodoNascimentoSousa.pdf. Acesso em: 20 jan. 2022.

STAKE, Robert. E. Pesquisa Qualitativa: estudando como as coisas funcionam. Porto Alegre: Penso, 2oIr.

VERGARA, Sylvia Constant. Projetos e Relatórios de Pesquisa em Administração. 13 ed. São Paulo, 20II. 